Southern Rhodesia, and M. G. Billing, Esq., Provincial Commissioner, the Secretariat, Lusaka. The conference was opened by Dr. Walter Adams, Principal of the University College of Rhodesia and Nyasaland.

The proceedings of the conference are available in roneographed form, price ros., edited and with an introduction by R. J. Apthorpe. Orders should be addressed to the Librarian, The Rhodes-Livingstone Institute, Lusaka, P.O. Box 900, as soon as possible. Also available from the Institute are: Proceedings of the 12 th Rhodes-Livingstone Institute Conference, September 1958-Social Relations in Industry, edited by D. Matthews and R. J. Apthorpe, I2s.; Proceedings of the IIth Rhodes-Livingstone Institute Conference-Present Interrelations in Central African Rural and Urban Life, edited by R. J. Apthorpe, 75. 6d. Proceedings of the I th, I 2 th, and I 3 th conferences may be purchased all together at $25 \mathrm{~s}$. a set, post free, for as long as stocks last, from the Librarian of the Institute.

(Communicated by R. J. Apthorpe)

\title{
Activités de l'IRSAC, 1958
}

Monsieur André Lux a terminé à Luluaborg une étude générale de la population congolaise et poursuit son plan de recherches axé sur les problèmes de l'intégration des Congolais de la province du Kasai à la vie économique moderne sous la forme du salaire rural et urbain. Il a achevé l'étude des chômeurs de Luluaborg et continue l'analyse du marché de l'emploi et de la consommation dans les agglomérations du Kasaï.

Au début de l'année 1958, le Ministre des Colonies et le Gouvernement Général chargèrent M. J. Jacobs d'élaborer un projet d'uniformisation de l'orthographie de la langue tetela, deuxième langue de la province du Kasaĩ après le tshiluba. Il existe en effet dans la région tetela deux orthographes différentes: l'une enseignée par les Missions catholiques, l'autre par les Missions méthodistes. Le projet d'orthographe unifiée, presenté par $M$. Jacobs à la séance du 9 septembre 1958 , de la Commission de Linguistique africaine, tient compte des suggestions des Africains et des Européens directement intéressés par la question. Au cours de cette même séance il fut décidé de la publication des ouvrages nécessaires à la réalisation de ce projet: un memorandum sur l'orthographe nouvelle, un manuel de textes et une liste orthographique.

\section{Nomination du Chef de Centre à Astrida}

Monsieur J. Vansins, chargé de fonctions de Chef de Centre de l'IRSAC à Astrida depuis le départ de M. J. J. Maquet, a été nommé Chef du Centre en titre le I er juillet 1958.

Licencié en Philosophie et Lettres (Section Histoire Moderne) de l'Université de Louvain, M. J. Vansina est arrivé au Congo en qualité de chercheur de l'IRSAC au début de i 953 et a travaillé durant son premier terme à l'histoire des institutions des Kuba, et à l'ethnologie de cette tribu. En 1957 il est chargé d'une étude sur l'histoire du Ruanda et de l'Urundi, dont le but principal est l'établissement d'un Manuel sur les bases d'une documentation orale. Ses recherches portent sur tous les aspects de l'histoire et notamment sur les migrations et l'évolution économique, sociale, politique et religieuse des deux pays. Elles s'efforcent de fixer un cadre chronologique précis basé sur de nouveaux documents. Le Dr R. Oliver, qui a fait un séjour au centre d'Astrida au mois de juillet 1958 , a visité en compagnie de M. Vansina et de l'Abbé Kagame, plusieurs anciennes capitales du Ruanda-Urundi, des tombeaux royaux et un ancien camp militaire au Ruanda. Au cours de ses entretiens avec M. Vansina il a été possible de fixer une chronologie absolue à l'histoire du Ruanda-Urundi en comparant les listes généalogiques du Buganda, Bunyoro, Nkore avec celles du Ruanda et de l'Urundi. Il est apparu également qu'une prospection archéologique des anciennes capitales du Ruanda-Urundi pourrait être fructueuse. 